\title{
AN INVERSION FORMULA FOR A KERNEL INVOLVING A MELLIN-BARNES TYPE INTEGRAL'
}

\section{R. K. SAXENA}

1. Introduction. Fox [5] has shown that with the help of fractional integration it is possible to establish the reciprocities which resemble,

$$
\begin{aligned}
& g(x)=\int_{0}^{\infty} k(u x) f(u) d u, \\
& f(x)=\int_{0}^{\infty} k(u x) g(u) d u,
\end{aligned}
$$

usually known as the Generalized Fourier Integral transform, closely although the functional equation satisfied by the kernel is much more general than

$$
K(s) K(1-s)=1,
$$

where $K(s)$ denotes the Mellin transform of the kernel $k(x)$. The main aim of the present paper is to find an inversion formula for an integral transform whose kernel $R_{p, q}(x)$ has a Mellin-Barnes type integral representation. On account of the general character of the kernel various integral transforms possessing Whittaker functions, Bessel functions, Meijer's $G$-functions etc. as kernels can be derived as particular cases. It has been shown by means of fractional integration theory that this kernel can be reduced to an exponential function and consequently the transform will reduce to Laplace transform which can be solved by known methods.

2. An integral transform. Let

$$
\begin{gathered}
R_{p, q}(x)=\frac{1}{2 \pi i} \int_{C} \chi(s) x^{-s} d s, \text { where } \\
\chi(s)=\frac{\prod_{j=1}^{p} \Gamma\left(\frac{a_{j}+s}{p}\right) \prod_{j=1}^{q} \Gamma\left(\frac{b_{j}+s}{m_{j}}\right)}{\prod_{j=1}^{q} \Gamma\left(\frac{c_{j}+s}{m_{j}}\right)} .
\end{gathered}
$$

Presented to the Society, December 12, 1965 under the title An inversion for $a$ kernel; received by the editors December 9, 1965.

1 This research is supported by a post-doctoral fellowship of the National Research Council of Canada. 
We now define an integral transform over the interval $(0, \infty)$ by the integral equation

$$
\phi(x)=\int_{0}^{\infty} R_{p, q}(x u) h(u) d u .
$$

The following are the conditions of validity of (6).

(i) $h(x)$ belongs to $L_{2}(0, \infty)$;

(ii) $x>0$;

(iii) $p$ is a positive integer and $q$ a nonnegative integer;

(iv) $m_{j}>0$ for $j=1,2, \cdots, q$;

(v) all the poles of the integrand in (4) are simple and must lie on the left of $C$;

(vi) the contour $C$ is a straight line parallel to the imaginary axis in the complex $s$-plane given by $s=1 / 2+i t, t$ being real and $-\infty<t$ $<\infty$;

(vii) $a_{i} \neq a_{j}, i \neq j, i=1,2, \cdots, p$. Similar conditions hold for all $b_{j}^{\prime}$ 's and $c_{j}^{\prime}$ 's, $j=1,2, \cdots, q$.

The function $R_{p, q}(x)$ will also be written as

$$
R_{p, q}\left(\begin{array}{l|c}
x & c_{1}, \cdots, c_{q} \\
a_{1}, \cdots, a_{p}, b_{1}, \cdots, b_{q}
\end{array}\right)
$$

wherever necessary for definiteness.

3. The Mellin transform. The Mellin transform of $h(z)$ will be denoted by $\mathfrak{M}\{h(z)\}$. If $\mathfrak{M}\{h(z)\}=H(s)$ we shall also write $h(z)$ $=\mathfrak{M}^{-1}\{H(s)\}$, where $\mathfrak{M}^{-1}$ indicates the inverse Mellin transform.

Formally we have

$$
\begin{aligned}
& H(s)=\mathfrak{M}\{h(u)\}=\int_{0}^{\infty} u^{s-1} h(u) d u, \\
& h(u)=\mathfrak{M}^{-1}\{H(s)\}=\frac{1}{2 \pi i} \int_{\sigma-i \infty}^{\sigma+i \infty} H(s) x^{-8} d s .
\end{aligned}
$$

We shall make use here of $L_{2}$-space theory in which simplest conditions are given. If

$$
h(u) \in L_{2}(0, \infty)
$$

and the 1.i.m. is with index 2 , then

$$
H(s)=\mathfrak{M}\{h(z)\}=\operatorname{lifim}_{N \rightarrow \infty} \int_{1 / N}^{N} h(z) z^{s-1} d z
$$


and also

$$
H(s) \in L_{2}(1 / 2-i \infty, 1 / 2+i \infty) \text {. }
$$

If

$$
H(s) \in L_{2}(1 / 2-i \infty, 1 / 2+i \infty)
$$

then

$$
h(z)=\mathfrak{M}^{-1}\{H(s)\}=\frac{1}{2 \pi i} \lim _{N \rightarrow \infty} \int_{1 / 2-i N}^{1 / 2+i N} H(s) z^{\bullet} d s
$$

and also $h(z) \in L_{2}(0, \infty)[9$, Theorem 71, p. 94$]$.

The following result will be found useful in our investigations given recently by Fox $[4$, p. 458].

Lemma. If (i) $x>0$ (ii) $h(z)$ and $g(z)$ both belong to $L_{2}(0, \infty)$ (iii) $\mathfrak{M}\{h(z)\}=H(s), \mathfrak{M}\{g(z)\}=G(s)$ and $G(s)$ is bounded on the line $s=1 / 2+i t,-\infty<t<\infty$, then

$$
\int_{0}^{\infty} g(x z) h(z) d z \in L_{2}(0, \infty)
$$

and

$$
\mathfrak{M}\left\{\int_{0}^{\infty} g(x z) h(z) d z\right\}=G(s) H(1-s),
$$

where the integrals of (12) and (13) are regarded as functions of $x$.

4. Lemma I. If (i) $x>0$ (ii) $a_{j} \geqq 0, j=1,2, \cdots, p$ (iii) $b_{j} \geqq 0$, $j=1,2, \cdots, q$ (iv) $h(x) \in L_{2}(0, \infty)$ then

$$
\int_{0}^{\infty} R_{p, q}(x u) h(u) d u \in L_{2}(0, \infty)
$$

and

$$
\mathfrak{M}\left\{\int_{0}^{\infty} R_{p, q}(x u) h(u) d u\right\}=\chi(s) H(1-s)
$$

where the left-hand side of (14) is regarded as a function of $x$.

Proof. In order to establish the above lemma, we must prove the following results.

(a) $R_{p, q}(z)$ belongs to $L_{2}(0, \infty)$.

(b) $\mathfrak{M}\left\{R_{p, q}(z)\right\}$ is bounded on the line $s=1 / 2+i t$. 
From the asymptotic expansion of $\Gamma(s+a)[12$, p. 279]

$$
\begin{aligned}
\log \Gamma(s+a)= & (s+a-1 / 2) \log s-s+1 / 2 \log 2 \pi \\
& +A s^{-1}+O\left(s^{-2}\right),
\end{aligned}
$$

where $O$ is the usual Landau order symbol and $|\operatorname{args}|<\pi-\delta, \delta>0$, it is evident that along the line $s=1 / 2+i t,-\infty<t<\infty$ for large positive and negative $t$,

$$
|\chi(s)|=\lambda t^{\mu} \exp \left(-\frac{1}{2} \pi|t|\right)\left[1+O\left(|t|^{-1}\right)\right],
$$

where $\lambda$ is a constant and

$$
\mu=1 / 2+1 / p \sum_{j=1}^{p} a_{j}-p / 2-\sum_{j=1}^{q}\left(b_{j}-c_{j}\right) / m_{j} .
$$

If $a_{j} \geqq 0, j=1,2, \cdots, p ; b_{j} \geqq 0, j=1,2, \cdots, q$ the singularity of the $s$-function on the right of (5) occurs only when $s$ is real and $s \leqq 0$. Hence from (16') it follows that $\chi(s)$ is bounded on the line $s=1 / 2+i t$ for all values of $t$ and it also belongs to $L_{2}(1 / 2-i \infty, 1 / 2+i \infty)$. From (11) it is now evident that $R_{p, q}(z)$ belongs to $L_{2}(0, \infty)$ and consequently

$$
\mathfrak{M}\left\{R_{p, q}(z)\right\}=\chi(s) .
$$

We are now justified in taking $g(z)=R_{p, q}(z)$ in the lemma of the preceding section and then after changing the variable of integration from $z$ to $u,(12)$ and (13) give rise to (14) and (15) respectively.

5. Fractional integration. Several definitions of fractional integration have been given by many workers including Erdélyi [2], Kober [6] and Weyl [11]. Erdélyi's definitions of fractional integration are slight variance of Kober's operators [6, p. 193].

Erdélyi [2] has given the following definitions of fractional integrations

(18) $\Omega(\alpha, \beta: m) h(x)=\frac{. m}{\Gamma(\alpha)} x^{\beta} \int_{x}^{\infty} u^{-\beta-m \alpha+m-1}\left(u^{m}-x^{m}\right)^{\alpha-1} h(u) d u$

and

$$
\Im(\alpha, \beta: m) h(x)=\frac{m}{\Gamma(\alpha)} x^{-\beta-m \alpha+m-1} \int_{0}^{x}\left(x^{m}-u^{m}\right)^{\alpha-1} u^{\beta} h(u) d u .
$$

The sufficient conditions of validity of (18) and (19) are

$$
\alpha>0, \beta>-1 / 2, m>0 \text { and } h(x) \in L_{2}(0, \infty) .
$$


If these conditions are satisfied, then

(21) $\Re(\alpha, \beta: m) h(x)$ and $\Im(\alpha, \beta: m) h(x)$ both belong to $L_{2}(0, \infty)$.

The following results $[2$, p. 222] are useful in our investigations.

$$
\begin{aligned}
\mathfrak{M}\{\mathfrak{R}(\alpha, \beta: m) h(x)\} & =\frac{\Gamma\left[\frac{(\beta+s)}{m}\right]}{\Gamma\left(\alpha+\frac{\beta+s}{m}\right)} \mathfrak{M}\{h(x)\}, \\
\mathfrak{M}\{\Im(\alpha, \beta: m) h(x)\} & =\frac{\Gamma[(\beta+1-s) / m]}{\Gamma\left(\alpha+\frac{\beta+1-s}{m}\right)} \mathfrak{M}\{h(x)\},
\end{aligned}
$$

provided that the conditions (20) hold.

Lemma II. If (i) $x>0$ (ii) $h(x) \in L_{2}(0, \infty)$ (iii) $\alpha \geqq 0$ (iv) $c_{j}>-1 / 2$ for $j=1,2, \cdots, q$ then

$$
\begin{aligned}
\Re\left(\alpha / m_{q}, c_{q}: m_{q}\right) & \int_{0}^{\infty} R_{p, q}(x u) h(u) d u \\
& =\int_{0}^{\infty} R_{p, q}\left(x u \mid \begin{array}{c}
c_{1}, \cdots, c_{q-1}, c_{q}+\alpha \\
a_{1}, \cdots, a_{p}, b_{1}, \cdots, b_{q}
\end{array}\right) h(u) d u
\end{aligned}
$$

where the integrals are considered as functions of $x$.

Proof. From Lemma I, the first integral of (24) belongs to $L_{2}(0, \infty)$ and so we can apply the operator $\Omega$ to it by (21). It is also evident that the left-hand side of (24) belongs to $L_{2}(0, \infty)$ and therefore the operator $\mathfrak{M}$ can be applied to it, by (9). Hence from (15) and (22) we obtain

$$
\begin{gathered}
\mathfrak{M}\left\{\Re\left(\alpha / m_{q}, c_{q}: m_{q}\right) \int_{0}^{\infty} R_{p, q}(x u) h(u) d u\right\} \\
=\frac{\Gamma\left(\frac{c_{q}+s}{m_{q}}\right)}{\Gamma\left(\frac{\alpha+s+c_{q}}{m_{q}}\right)} \chi(s) H(1-s) \\
\quad=\mathfrak{M}\left\{\int_{0}^{\infty} R_{p, q}\left(x u \mid \begin{array}{c}
c_{1}, \cdots, c_{q-1}, c_{q}+\alpha \\
a_{1}, \cdots, a_{p}, b_{1}, \cdots, b_{q}
\end{array}\right) h(u) d u\right\} .
\end{gathered}
$$


From (14) and (21) the functions in (26) operated upon by $\mathfrak{M}$ both belong to $L_{2}(0, \infty)$. Hence from (10) each side of (26) belongs to $L_{2}(1 / 2-i \infty, 1 / 2+i \infty)$ and so from (11) the operator $\mathfrak{M}^{-1}$ can be applied to (26). This application finally establishes (24) and completes the proof of Lemma II.

\section{Inversion formula.}

TheOREM I. If (i) $b_{j}-c_{j} \geqq 0, j=1,2, \cdots, q$ (ii) $c_{j}>-1 / 2, j$ $=1,2, \cdots, q$ (iii) $x>0$ (iv) $a_{k}+k-1 \geqq 0$ for $k=1,2, \cdots, p$ (v) $h(x)$ is a solution of

$$
\int_{0}^{\infty} R_{p, q}(x u) h(u) d u=\phi(x),
$$

which belongs to $L_{2}(0, \infty)$ then

$$
\begin{aligned}
h(x)=p^{-1 / 2}(2 \pi)^{1 / 2-p / 2} \mathcal{L}^{-1}\left\{\prod_{k=1}^{p}\right. & \Re\left(\frac{a_{k}+k-1}{p}, k-1: p\right) \\
& \left.\times \prod_{j=1}^{q} \Omega\left(\frac{b_{j}-c_{j}}{m_{j}}, c_{j}: m_{j}\right) \phi\left(\frac{x}{p}\right)\right\}
\end{aligned}
$$

where $\mathfrak{L}^{-1}$ denotes the inverse Laplace transform.

Proof. From condition (v) and (14) it is observed that both sides of (27) belong to $L_{2}(0, \infty)$. The operator

$$
\Re\left(\frac{b_{q}-c_{q}}{m_{q}}, c_{q}: m_{q}\right)
$$

can therefore be applied by virtue of (20) and (21). Hence from (24) it follows that

$$
\begin{aligned}
\int_{0}^{\infty} R_{p, q-1}\left(\left.x u\right|_{a_{1}, \cdots, a_{p}, b_{1}, \cdots, b_{q-1}}\right) h(u) d u \\
=\Omega\left\{\left(b_{q}-c_{q}\right) / m_{q}, c_{q}: m_{q}\right\} \phi(x) .
\end{aligned}
$$

The above integral equation may be regarded as a reduction formula for the integral in (27). Applying the operator $\Re$ successively to (29) with $b_{q}$ replaced by $b_{q-1}, b_{q-2}, \cdots, b_{1} ; c_{q}$ replaced by $c_{q-1}$, $c_{q-2}, \cdots, c_{1} ; m_{q}$ replaced by $m_{q-1}, m_{q-2}, \cdots, m_{1}$ and then $b_{q}$ replaced by $a_{p}, a_{p-1}, \cdots, a_{1} ; c_{q}$ replaced by $p-1, p-2, \cdots, 1,0$ with $m_{q}=p$ and applying the Legendre's multiplication formula for the Gamma functions $[3$, p. 6$]$ we find that 


$$
\begin{aligned}
& (2 \pi)^{p / 2-1 / 2} p^{1 / 2} \int_{0}^{\infty} e^{-p x u} h(u) d u \\
& \quad=\left\{\prod_{k=1}^{p} \Re\left(\frac{a_{k}+k-1}{p}, k-1: p\right) \prod_{j=1}^{q} \Re\left(\frac{b_{j}-c_{j}}{m_{j}}, c_{j}: m_{j}\right) \phi(x)\right\}
\end{aligned}
$$

which is equivalent to (28).

COROLlaRY 1 . When $q=0,(28)$ reduces to a result given quite recently by Fox $[4$, p. 466].

CoRollary 2. For $p=m_{j}(j=1,2, \cdots, p)=1, R_{p, q}(x)$ reduces to a Meijer's G-function [3, p. 207] and the theorem yields the following interesting result:

$$
\text { If (i) } b_{j}-c_{j} \geqq 0, j=1,2, \cdots, q \text { (ii) } c_{i}>-1 / 2 \text { for } j=1,2, \cdots, q
$$
(iii) $x>0$ (iv) $a_{1} \geqq 0$ and (v) $h(x)$ is a solution of the integral equation

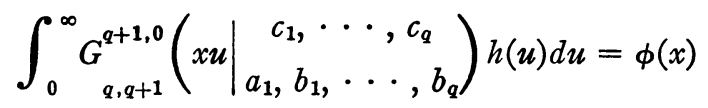

which belongs to $L_{2}(0, \infty)$ then

$$
h(x)=\mathfrak{L}^{-1}\left\{\Re\left(a_{1}, 0: 1\right) \prod_{j=1}^{q} \Re\left(b_{j}-c_{j}, c_{j}: 1\right) \phi(x)\right\} .
$$

The integral equation (30) have been studied by Bhise [1].

It is interesting to note that the inversion formulae for Meijer and Varma transforms [7] and [10] can be easily deduced from Corollary 2 by giving suitable values to its parameters. The inversion formula for the Varma transform has been given by the author in an earlier paper [8].

7. A generalization of the kernel $R_{p, q}(x)$. In this section we consider briefly the inversion formula for the integral transform defined by the integral equation

$$
\int_{0}^{\infty} R_{p, q, r}(x u) h(u) d u=\phi(x),
$$

which is more general than (6) and

$$
R_{p, q, r}(x)=\frac{1}{2 \pi i} \int_{C^{\prime}} \zeta(s) x^{-s} d s,
$$

where 


$$
\zeta(s)=\frac{\prod_{j=1}^{p} \Gamma\left(\frac{a_{j}+s}{p}\right) \prod_{j=1}^{q} \Gamma\left(\frac{b_{j}+s}{m_{j}}\right) \prod_{j=1}^{r} \Gamma\left(\frac{1+d_{j}-s}{n_{j}}\right)}{\prod_{j=1}^{q} \Gamma\left(\frac{c_{j}+s}{m_{j}}\right) \prod_{j=1}^{r} \Gamma\left(\frac{1+e_{j}-s}{n_{j}}\right)}
$$

$n_{j}>0$ for $j=1,2, \cdots, r$ and the contour $C^{\prime}$ is a straight line parallel to the imaginary axis in the $s$-plane given by $s=1 / 2+i t,-\infty<t<\infty$ and all the poles of $\Gamma\left(\left(a_{j}+s / p\right)\right.$ for $j=1,2, \cdots, p$ and $\Gamma\left(\left(b_{j}+s\right) / m_{j}\right)$ for $j=1,2, \cdots, q$ must lie to the left of $C^{\prime}$ while those of $\Gamma\left(\left(1+d_{j}\right.\right.$ $-s) / n_{j}$ ) for $j=1,2, \cdots, r$ to the right of it. The other restrictions on the parameters are same as in $\$ 2$.

Theorem II. If (i) $b_{j}-c_{j} \geqq 0, j=1,2, \cdots, q$ (ii) $c_{j}>-1 / 2$ for $j=1,2, \cdots, q$ (iii) $d_{j}-e_{j} \geqq 0$ for $j=1,2, \cdots, r$ (iv) $e_{j}>-1 / 2$ for $j=1,2, \cdots, r$ (v) $x>0$ (vi) $a_{k}+k-1 \geqq 0$ for $k=1,2, \cdots, p$ (vii) $h(x)$ is a solution of

$$
\int_{0}^{\infty} R_{p, q, r}(x u) h(u) d u=\phi(x)
$$

which belongs to $L_{2}(0, \infty)$ then

$$
h(x)=p^{-1 / 2}(2 \pi)^{1 / 2-p / 2} \mathcal{L}^{-1}\left\{\prod_{k=1}^{p} \Omega\left(\frac{a_{k}+k-1}{p}, k-1: p\right)\right.
$$

$$
\left.\times \prod_{j=1}^{q} \Omega\left(\frac{b_{j}-c_{j}}{m_{j}}, c_{j}: m_{j}\right) \prod_{l=1}^{r} \Im\left(\frac{d_{l}-e_{l}}{n_{l}}, e_{l}: n_{l}\right) \phi(x / p)\right\} .
$$

The proof of the above theorem can be developed on similar lines as in the preceding section by employing the operator $\mathfrak{T}$ also and using a lemma corresponding to Lemma II for the operator $\Omega$ which can be established in the same way by means of (15) and (23).

The author is extremely grateful to Professor Charles Fox for giving some helpful suggestions in the preparation of this paper.

\section{REFERENCES}

1. V. M. Bhise, Inversion formulae for a generalized Laplace integral, J. Vikram Univ. India 3 (1959), 57-63.

2. A. Erdélyi, On some functional transformations, Univ. e Politec. Torino Rend. Sem. Mat. 10 (1940), 217-234.

3. A. Erdélyi, et al, Higher transcendental functions, Vol. 1, McGraw-Hill, New York, 1953.

4. C. Fox, $A n$ inversion formula for the kernel $K_{\mathrm{v}}(x)$, Proc. Cambridge Philos. Soc. 61 (1965), 457-467. 
5. - Integral transformations based upon fractional integration Proc. Cambridge Philos. Soc. 59 (1963), 63-71.

6. H. Kober, On fractional integrals and derivatives, Quart. J. Math. Oxford Ser. 11 (1940), 193-211.

7. C. S. Meijer, Eine neue Erweiterung der Laplace Transformation I, Nederl. Akad. Wetensch. Proc. 44 (1941), 727-737; 831-839.

8. R. K. Saxena, An inversion formula for the Varma transform, Proc. Cambridge Philos. Soc. 62 (1966) (to appear).

9. E. C. Titchmarsh, An introduction to the theory of Fourier integrals, Oxford, 1939.

10. R. S. Varma, On a generalization of Laplace integral, Proc. Nat. Acad. Sci. India 20 (1951), 209-216.

11. H. Weyl, Vierteljschr. Naturforsch. Ges. Zürich 62 (1917), 296-302.

12. E. T. Whittaker and G. N. Watson, $A$ course of modern analysis, Cambridge, 1962.

MCGILL UNIVERSITY 